\title{
Semantic Behavior of Indexed Co-inductive Data Type in Fibrational Setting
}

\author{
Miao De-cheng ${ }^{1}$, Xi Jian-qing ${ }^{2}$, Jiang Chang-jin ${ }^{1}$ and Liang Yong-lin ${ }^{1}$ \\ ${ }^{1}$ School of Information Science and Engineering, Shaoguan University, Shaoguan \\ Guangdong 512005, China \\ ${ }^{2}$ School of Software, South China University of Technology, Guangzhou Guangdong \\ 510640, China \\ tony10860@126.com
}

\begin{abstract}
Traditional methods such as category theory and coalgebra have some drawbacks to analyze semantic behavior of indexed co-inductive data type. Aiming at the problem above, this paper explored indexed co-inductive data type in programming by Fibrations theory. Our main work was that we firstly made some basic logical structures of indexed co-inductive data type over a fibration such as truth and quotient functor; using endo-functor in base categories and their equation-preserving lifting in total categories, then we analyzed semantic behavior of indexed co-inductive data type; at last we briefly introduced applications of Fibrations theory on indexed co-inductive data type by example. Compared with traditional methods, brief descriptions and flexible expansibility of Fibrations theory can analyze semantic behavior of indexed co-inductive data type accurately, and superior abstractness of Fibrations theory doesn't rely on particular computing environments to compute semantics.
\end{abstract}

Keywords: Semantic Behavior, Coinduction rule, Fibrations theory, Lifting, Co-recursive operation

\section{Introduction}

As the dual concepts of inductive data type, co-inductive data type [1] is a new method to study semantic behavior of data type in programming and type theory. Co-inductive data type whose math basis is coalgebra [2-3] observes dynamic behavior of program languages from outside, it provides a complementary method for inductive data type to enhance syntax construction and semantic computation of programming. Indexed co-inductive data type is a co-inductive data type whose ability of semantic computing is stronger; it can deal with more complex data structure. At present indexed co-inductive data type has become an important component and emphasis. Traditional methods including category theory and coalgebra make model in local Cartesian closed category, indexed co-inductive data type and relation category describing its semantic behavior coexist the same category, which results in the functor and its lifting are identical, so there exists some limitation in analyzing semantic behavior.

Fibrations theory is an emerging trend in computer science recently, especially in categorical methods, it has many applications in database system modelling [4-5], software specifications [6] and programming [7-8]. It provides a general thinking methodology, research means and theory tools for describing structured relationships between formal systems effectively.

In the formal framework of Fibrations theory, the relation describing semantic behavior of indexed co-inductive data type doesn't limit to function or morphism any more, but lifts to objects in total category. More importantly, indexed co-inductive data type and relation category describing its semantic behavior do not coexist in the same category, but 
constructing functor lifted in total category to depict abstractly semantic computing and program logics of indexed co-inductive data type, Hermida and Jacobs did lots of works in this field [9].

In this paper, we applying Fibrations theory to research indexed co-inductive data type, by taking indexed co-inductive data type to be object set in base category, taking their semantic behavior to be object set in total category firstly; then we establish the responsible relation in program logic directly between indexed co-inductive data type and it semantic behavior by equation functor and quotient functor.

Our primary works are studying semantic behavior of indexed co-inductive data type and its co-inductive rule by Fibrations theory. The rest of this paper is structured as follows. In Section 2, we firstly introduced some basic concepts needed for our works, such as Cartesian arrow and fibration. In Section 3, we presented indexed fibration et al. based on slice category to analyze semantic behavior of indexed co-inductive data type, and introduced the applications of Fibrations theory briefly by example. Then we researched some related works in the field of co-inductive data type currently in Section 4. At last, we summarized our conclusions and discussed our future works.

\section{Fibration and Op-Fibration}

\subsection{Fibration and Re-Indexed Functor}

We assume readers have some categorical foundation including ad joint functor, pullback and terminal object. Considering not presupposing the set theoretical models based on mathematical logic, currently some basic math literatures don't require all morphisms to form a set when they define category; but analyzing from the practical applications perspective of discrete objects processed in computer science, we deem it is reasonable to confine all morphisms to a set. If all objects and morphisms can form two sets respectively in a category, the category is called to be a small category [10]. All research objects in this paper are based on small category, more details about Fibrations theory can be found in [10-12]. For category $C$, let $\boldsymbol{O}_{b j} C$ to be set of objects and $\boldsymbol{M o r}_{\mathrm{o}} \mathrm{C}$ to be set of morphisms. We introduce some basic concepts in this paper.

Definition 1. Let $P: T \rightarrow B$ be a functor between two small categories $T$ and $B, f: C \rightarrow D \in M$ or $B, P(Y)=D$. A morphism $u: X \rightarrow Y \in M$ orT is a Cartesian arrow of $f$ and $Y$, if $P(u)=f, \forall v: Z \rightarrow Y \in M$ orT and $\forall h: P(Z) \rightarrow C \in M$ or $B$, it satisfies diagram commutes, that is, $f \circ h=P(v)$. There also exists an unique $w: Z \rightarrow X \in M$ orT such that $u^{\circ} w=v$ and $P(w)=h$.

For Cartesian arrow $u$ of $f$ and $Y$, we say $u$ lies above $f$; similarly, $Y$ lies above $D$. If $u$ is a cone [10] in category $T$, the Cartesian arrow $u$ in Definition 1 is also a universal cone in $T$ by the uniqueness of cones morphism $w$, namely, limit cone. Accordingly, the vertex $x$ of universal cone $u$ is the terminal object of $x$ [13], while Cartesian arrow $u$ is an isomorphism by universal properties of universal cones. We write $f_{Y}^{\downarrow}$ for Cartesian arrow $u$ of $f$ and $Y$ in Definition 1 for simplification.

Definition 2. Let $P: T \rightarrow B$ be a functor between two small categories $T$ and $B$. If there exists a Cartesian arrow $f_{Y}^{\downarrow}$ of $f$ and $Y$ for $\forall Y \in \boldsymbol{O}_{b j} T$ and $\forall f: C \rightarrow P(Y) \in \boldsymbol{M}$ or $B$, then we call $P$ a fibration.

By Definition 2, a fibration is a functor that ensures a large of Cartesian arrows. For a fibration $P: T \rightarrow B$, we call $B$ the base category and $T$ the total category of $P$. For an object $C$ in $\boldsymbol{o b j j}_{B}, \exists X \in \boldsymbol{O}_{b j T}, k \in \boldsymbol{M} o r T$, if it satisfies $P(X)=C$ and $P(k)=i d_{c}$, then the 
subcategory $T_{c}$ constituted by $x$ and $k$ is called to be a fiber over $C$ in [10], and $k$ is a vertical morphism. In fact, fiber $T_{C}$ is a full subcategory of total category $T$.

Write $f^{*}(Y)$ for domain of Cartesian arrow $f_{y}^{\downarrow}$, then $f^{*}(Y)$ lies over $C$, that is, $Y \in \boldsymbol{O} \operatorname{bj} T_{D}$, $f^{*}(Y) \in \boldsymbol{O} b j T_{c}$. Therefore we have the definition of re-indexed functor.

Definition 3. If a morphism $f: C \rightarrow D$ in base category $B$ is extended to be a functor $f^{*}: T_{D} \rightarrow T_{C}$ between fibers $T_{D}$ and $T_{C}$, we call $f^{*}$ a re-indexed functor induced by $f$.

Morphism $f$ is the relationship between indexed co-inductive data types in base category, and re-indexed functor $f^{*}$ is a lifting of $f$ in total category, which is related to their semantic behaviors. The dual concept to fibration, i.e., op-fibration, is as follows.

\subsection{Op-Fibration and Opposite Re-indexed Functor}

Definition 4. Let $P: T \rightarrow B$ be a functor between two small categories $T$ and $B$, $f: C \rightarrow D \in \operatorname{Mor} B, u: X \rightarrow Y \in M$ ort . The morphism $u$ is called to be an opposite Cartesian arrow of $f$ and $X$ if $P(X)=C, P(u)=f, \forall v: X \rightarrow Z \in M$ orT $\quad$ and $\forall h: D \rightarrow P(Z) \in M$ or $B$. It satisfies diagram commuting, that is, $h \circ f=P(v)$, and there exists an unique $w: Y \rightarrow Z \in M$ orT such that $w^{\circ} u=v$ and $P(w)=h$.

Similar to Definition 1, if $u$ is a co-cone [10] in category $T$, the opposite Cartesian arrow $u$ in Definition 4 is a universal co-cone in $T$ by the uniqueness of co-cones morphism $w$, namely, co-limit co-cone. Accordingly, the vertex $Y$ of universal co-cone $u$ is the initial object of $u$ in [13], while opposite Cartesian arrow $u$ is an isomorphism by universal properties of universal co-cones.

Definition 5. Let $P: T \rightarrow B$ be a functor between two small categories $T$ and $B$. If for $\forall X \in \boldsymbol{O} b_{j} T$ and $\forall f: P(X) \rightarrow D \in M$ or $B$, there exists an opposite Cartesian arrow of $f$ and $X$, then we call $P$ an op-fibration.

Definition 6. If a functor $P: T \rightarrow B$ between two small categories $T$ and $B$ is simultaneously a fibration and an op-fibration, then it is a bifibration.

Without loss of generality, we write $f_{\downarrow}^{X}$ for the opposite Cartesian arrow $u$ of $f$ and $x$ in definition 4, let " $f(x)$ be the codomain of $f_{\downarrow}^{X}$, then we say " $f(X)$ lies above $D$, i.e., $X \in \boldsymbol{O} b j T_{C}, " f(X) \in \boldsymbol{O} b j T_{D}$.

Definition 7. A morphism $f: C \rightarrow D$ in base category $B$ is extended to be a functor ${ }^{*}: T_{C} \rightarrow T_{D}$ between fibers $T_{c}$ and $T_{D}$, we call ${ }_{f}$ is an opposite re-indexed functor induced by $f$.

\subsection{Adjunction Property of Re-indexed and Op- re-indexed Functor}

Definition 8. If $F \dashv G: C \rightarrow D$ is a pair of ad joint functors, $\eta, \varepsilon$ is the unit and co-unit of this adjunction, respectively, and for any two objects, namely, $\forall X \in \boldsymbol{O} b j C, \forall Y \in \boldsymbol{O} b j D$, $\exists f: F(X) \rightarrow Y \in M$ or $D, \exists g: X \rightarrow G(Y) \in M$ orC , then the transpose of $f$ and $g$ is $G(f) \eta_{X}$ and $\varepsilon_{Y} F(g)$ respectively.

Theorem 1. Let $P: T \rightarrow B$ be a fibration between two small categories $T$ and $B$. Then $P$ is a bifibration iff $\forall f: C \rightarrow D \in M$ or $B$, the re-indexed functor $f^{*}$ has a left ad joint functor " ${ }_{f}$ which is an opposite re-indexed functor. 
Proof. $\Rightarrow$. Let ${ }_{f} \dashv f^{*}: T_{C} \rightarrow T_{D}$ be a pair of ad joint functors, the unit be $\eta$, co-unit be $\varepsilon$, and $P: T \rightarrow B$ be a fibration between two small categories $T$ and $B$. For $\exists Y \in \boldsymbol{O}_{b j} T_{D}$, we can construct a Cartesian arrow $f_{Y}^{\downarrow}: f^{*}(Y) \rightarrow Y$ whose codomain is $Y . \exists X \in \boldsymbol{O} b j T_{c}$, let $l: X \rightarrow{ }^{\circ} f(X)$ be morphism above $f$, next we prove that $l$ is an opposite Cartesian arrow above $f$. It satisfies $l=f_{w_{f}(X)}^{\downarrow}{ }^{\circ} \eta_{x}$ by the adjunction property of ${ }^{*} f \dashv f^{*}$, seen from Figure 1. We write $i d$ for identify morphism, if $g: X \rightarrow Y$ is another morphism above $f$, let $\phi: X \rightarrow f^{*}(Y)$ be vertical morphism in $T_{c}$, we have $P(\phi)=i d_{c}$. By definition 1 we obtain that $g=f_{Y}^{\downarrow} \circ \phi$, Cartesian arrow $f_{Y}^{\downarrow}$ is a universal cone, whose universal property ensures $\phi$ is the unique morphism from $g$ to $f_{Y}^{\downarrow}$. We write $\hat{\phi}$ for the transpose of $\phi$ under the adjunction ${ }^{*} f \dashv f^{*}$, then $\hat{\phi}=\varepsilon_{Y}{ }^{\circ}{ }^{*} f(\phi):{ }^{\prime} f(X) \rightarrow Y$, and $f^{*}(\hat{\phi})^{\circ} \eta_{X}=\phi$. Universal property of universal cone $f_{Y}^{\downarrow}$ ensures the unique existence of $f^{*}(\hat{\phi})$, and it satisfies diagram commuting, that is, $\hat{\phi}^{\circ} f_{f_{f(x)}}^{\downarrow}=f_{y}^{\downarrow} \circ f^{*}(\hat{\phi})$. Above all, there exist equations, namely, $\hat{\phi^{\circ} l}=\hat{\phi} \circ f^{\downarrow}{ }^{\downarrow}(X){ }^{\circ} \eta_{X}=f_{Y}^{\downarrow} \circ f^{*}(\hat{\phi}) \circ \eta_{X}=f_{Y}^{\downarrow} \circ \phi=g$, that is, $g=\hat{\phi}^{\circ} l$, then the transpose $\hat{\phi}$ of $\phi$ is the unique morphism from $l$ to $g$, and $P(\hat{\phi})=i d_{0}$. We thus prove that $l$ is an opposite Cartesian arrow $f_{\downarrow}{ }^{x}$ above $f$ by Definition 4 .

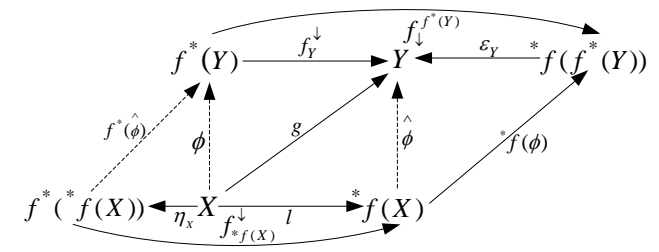

\section{Figure 1. Proof of Opposite Cartesian Arrow}

$\Leftarrow$. We assume $g: X \rightarrow Y \in M$ or $T$ lies above $f$, write $T_{c}\left(X, f^{*}(Y)\right)$ for the set composed of morphisms above $C$ in fiber $\left.T_{C}, T_{D} i f(X), Y\right)$ for the set composed of morphisms above $D$ in fiber $T_{D}$. For $\forall k: X^{\prime} \rightarrow X \in \operatorname{Mor}_{C}, \forall h: Y \rightarrow Y^{\prime} \in \operatorname{Mor} T_{D}$, because $P: T \rightarrow B$ is a bifibration, it give us an one-to-one corresponding map $\varphi_{x, Y}: T_{D}\left({ }^{*} f(X), Y\right) \rightarrow T_{c}\left(X, f^{*}(Y)\right)$. We write $k^{o p}: X \rightarrow X^{\prime} \in \operatorname{MorT}_{c}$ for an opposite morphism of $k$, it satisfies that $k^{o p} \circ f_{\downarrow}^{X o p}=f_{\downarrow}{ }^{\prime} o p \circ{ }^{*} f\left(k^{o p}\right)$ and $i d_{f^{*}(Y)}{ }^{\circ} f_{Y}^{\downarrow o p}=f_{Y}^{\downarrow o p} \circ i d_{Y}$, so the left part of diagram in Figure 2 commutes. Similarly, it also satisfies $i d_{X}{ }^{\circ} f_{\downarrow}^{X o p}=f_{\downarrow}^{X o p} \circ_{i d d_{* f(X)}}$ and $f^{*}(h)^{\circ} f_{Y}^{\downarrow o p}=f_{Y}^{\downarrow o p}$, i.e., the right part of diagram in Figure 2 also commutes, so $\varphi_{x, Y}$ is a natural isomorphism. We thus prove ${ }^{*} f_{f} f^{*}$ by definition of ad joint functors in [13]. 


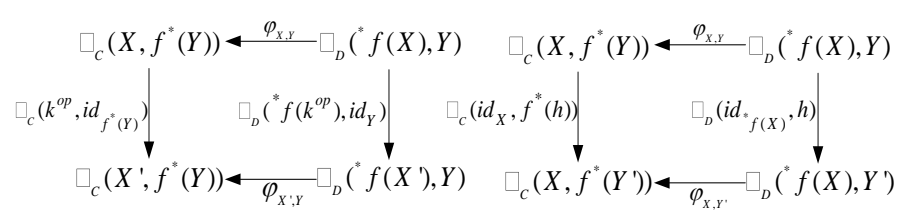

Figure 2. Proof of Adjunction Property

Theorem 1 gives a condition for determining if a fibration is a bifibration. At the same time, it also combines the adjunction property of re-indexed functor $f^{*}$ and its opposite re-indexed functor ${ }^{*} f$ in the framework of Fibration theory well.

\section{Semantic Property of Indexed Co-inductive Data Type}

From the viewpoint of Fibrations theory, indexed co-inductive data type is a common coinductive data type with discrete indexed objects such as streams, lists and trees. Based on the results from literature [14] and [15], this section constructs indexed fibration by Fibrations theory to analyze semantic behavior of indexed co-inductive data type.

\subsection{Truth Functor and Relation Fibration}

Definition 9. Let $P: T \rightarrow B$ be a fibration between two small categories $T$ and $B$. For $\forall D \in O b j B$. If $\exists \mathbf{1}_{D} \in \boldsymbol{O}_{b j T_{D}}$ is a terminal object in fiber $T_{D}$, and for $\forall f: C \rightarrow D \in \operatorname{Mor} B, f^{*}\left(\mathbf{1}_{D}\right)$ is a terminal object in fiber $T_{c}$, i.e., the re-indexed functor $f^{*}$ preserves terminal objects, then we call fibration $P$ has fibered terminal objects.

Definition 10. Let $P: T \rightarrow B$ be a fibration between two small categories $T$ and $B$, and functor $T_{P}: B \rightarrow T$ maps $\forall C \in \boldsymbol{O} b j B$ to a terminal object in fiber $T_{C}$. Then $T_{P}$ is a truth functor of fibration $P$. If $T_{P}$ has a right ad joint functor $\{-\}$, then $\{-\}$ is a comprehension functor of $P$.

Let $\mathbf{1}_{B}$ and $\mathbf{1}_{T}$ be the terminal object of base category $B$ and total category $T$ respectively. Then $P\left(\mathbf{1}_{T}\right)=\mathbf{1}_{B}$. For $\forall C \in \boldsymbol{O}$ bj $B$, there exists an unique morphism $u: C \rightarrow \mathbf{1}_{B}$ such that $T_{P}(C) \cong u^{*}\left(\mathbf{1}_{T}\right)$. For $\forall f: C \rightarrow D \in \boldsymbol{M}$ or $B, f^{*}\left(T_{P}(D)\right) \cong T_{P}(C)$, truth functor $T_{P}$ maps $f$ to its Cartesian arrow $f_{T_{,}(D)}^{\downarrow}$ in total category $T$.

Definition 11. Let $P: T \rightarrow B$ be a fibration between two small categories $T$ and $B$, and its base category $B$ has products. Let $\Delta: B \rightarrow B$ be a diagonal endo-functor above $B$, it maps $\forall C \in O b j B$ to product object $C \times C$. The pullback of $P$ along $\Delta$ constructs fibration $\operatorname{Rel}(P): \operatorname{Rel}(T) \rightarrow B$, and $\operatorname{Rel}(P)$ is a relation fibration of $P$.

The object of total category $\operatorname{Rel}(T)$ on $\operatorname{Rel}(P)$ is relation $(C, D)$. For another object $\left(C^{\prime}, D^{\prime}\right)$ in $\operatorname{Rel}(T)$, let $f: C \rightarrow C^{\prime}$ and $g: D \rightarrow D^{\prime}$ be two morphisms. Then $(f, g):(C, D) \rightarrow\left(C^{\prime}, D^{\prime}\right) \in M \operatorname{or} \operatorname{Rel}(T)$. The relation fibration $\operatorname{Rel}(P)$ in Figure 3 maps relation $(C, D)$ to object $C$ in base category $B$, functor $\Pi$ maps $(C, D)$ to object $D \operatorname{in} T$, and $P(D)=\Delta(C)$. At the same time, property pullback-preserving of Definition 11 ensures that fiber $\operatorname{Rel}(T)_{C}$ above $C$ on $\operatorname{Rel}(P)$ is isomorphism up to fiber $T_{C \times C}$ above $C \times C$ on $P$, i.e., $\operatorname{Rel}(T)_{C} \cong T_{C \times C}$. 


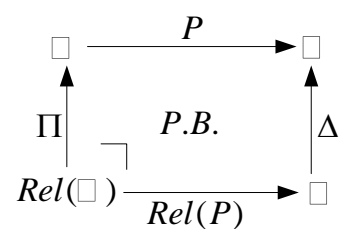

Figure 3. Relation Fibration $\operatorname{Rel}(P)_{\text {for }}$ f

The process of constructing a new fibration by given fibration is change of base. For example, we construct $\operatorname{Rel}(P)$ by change of base in Definition 11 from $P$. Change of base preserves structure, such as preserves fibered terminal objects [13], that is, if $P$ has truth functor $T_{P}$, then $\operatorname{Rel}(P)$ has truth functor $T_{\operatorname{Rel}(P)}$, and $T_{\operatorname{Rel}(P)}(C)=T_{P}(C \times C)$.

Theorem 2. Let $P: T \rightarrow B$ be a bifibration between two small categories $T$ and $B$, base category $B$ has pullbacks. If for each pullback square in $B$, natural transformation ${ }^{\prime}{ }^{\circ}{ }^{*} \rightarrow g^{*} \circ{ }^{*}$ is an isomorphism, then $P$ satisfies Beck-Chevalley condition.

Proof. Let $\eta_{f}$ be unit of ad joint functor ${ }_{f} \dashv f^{*}$, and $\varepsilon_{s}$ be co-unit of ad joint functor $s^{\prime} \dashv s^{\prime}$ (Figure 4). Then $\eta_{f}=I d_{T_{B}}, \varepsilon_{s}=I d_{T_{C}}$. The following equation holds, i.e., $\left(s^{\circ} t^{\circ}\right)^{\circ} \eta_{f}=\left(s^{\circ} t^{\circ}\right)^{\circ}\left(f^{*} \circ^{\circ} f\right)$, the pullback square in Figure 4 satisfies diagram commuting, namely, $f \circ t=g{ }^{\circ} s$, and $s$ is pullback of $f$ along $g, t$ is pullback of $g$ along $f$, by the pullback property of re-indexed functor, we have the isomorphism expression $t^{\circ} \circ f^{*} \cong s^{\circ} \circ g^{*}$. So we get the following equations, i.e., $\left(s^{\circ} t^{\circ}\right) \circ\left(f^{\circ} \circ^{\circ} f\right)=s^{\circ}\left(\quad t^{\circ} \circ f^{*}\right) 0^{*} f \quad \cong s^{\circ}\left(s^{\circ} \circ g{ }^{*}\right) 0^{\circ} f \quad$ and

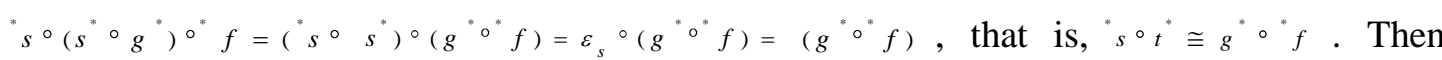
natural transformation ${ }^{*} s^{\circ} t^{*} \rightarrow g^{*}{ }^{*}{ }^{*} f$ is an isomorphism.

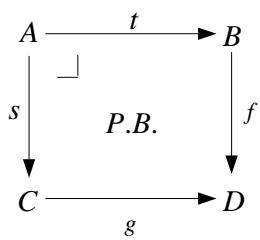

Figure 4. A Pullback Square in Base Category ${ }_{B}$

In another words, based on pullback square in base category on a bifibration $P$, Theorem 2 defines a natural transformation, whose functors preserve structure between corresponding fibers in total category $T$. The theorem further ensures re-indexed functor and its opposite reindexed functor satisfy appropriate properties of diagram commuting.

Definition 12. Let $P: T \rightarrow B$ be a bifibration satisfying Beck-Chevalley condition, base category $B$ has products, and $T_{P}$ be the truth functor of $P$. For $\forall C \in \boldsymbol{O} b j B$, the action function $\delta_{C}: C \rightarrow C \times C$ of natural transformation $\delta: I d_{B} \rightarrow \Delta$ at $C$ extends an opposite reindexed functor ${ }^{*} \delta, E q_{P}: B \rightarrow \operatorname{Rel}(T)$ is an equation functor of $P$, and $E q_{P}={ }^{*} \delta{ }^{\circ} T_{P}$.

Truth functor $T_{P}$ maps $C$ to the terminal object $T_{P}(C)$ in $T_{C}$, by Definition $11 \operatorname{Rel}(P)$ is the change of base of $P$ along $\Delta$. If $P$ has fibered terminal object, then $\operatorname{Rel}(P)$ also has fibered terminal object. The opposite re-indexed functor ${ }^{*} \delta$ in Definition 12 maps $T_{P}(C)$ to $" \delta\left(T_{P}(C)\right)$, and $" \delta\left(T_{P}(C)\right) \in \boldsymbol{O} b j\left(T_{C \times C} \cong \operatorname{Rel}(T)_{C}\right)$, equation functor $E q_{P}$ of $P$ maps 
$\forall f \in M$ or $B$ to the unique morphism above $f \times f$ determined by $\delta_{f}$ and $\left(\delta_{C}\right)_{\downarrow}^{T_{f}(c)}$. The intuitional meaning of equation functor is that same parameter has same results in [9].

\subsection{Indexed Fibration and Its Equation Functor}

Theorem 3. Let $P: T \rightarrow B$ be a fibration or bifibration between two small categories $T$ and $B$, and $T_{P}: B \rightarrow T$ be a truth functor of $P$. There $\exists I \in O \operatorname{Obj} B$, where $I$ is a discrete indexed object in base category $B$. Let indexed functor $P / I: T / T_{P}(I) \rightarrow B / I$ be $P / I(u)=P(u): P(Y) \rightarrow I \in \boldsymbol{O} b j B / I$ for $\forall u: Y \rightarrow T_{P}(I) \in \boldsymbol{O} b j T / T_{P}(I)$. Then the indexed functor $P / I$ is also a fibration or bifibration.

Proof. For $\forall f: C \rightarrow D \in M$ or $B$, there exists a Cartesian arrow $f_{x}^{\downarrow}: f^{\natural}(X) \rightarrow X$ above $f$ on fibration $P$ satisfying $P(X)=D$. And there also exists a unique morphism $w: T_{P}(I) \rightarrow f^{*}(X)$ such that $v=f_{x}^{\downarrow} \circ w$ and $P(v)=f \circ h$ (Fig.5). Let $\alpha: D \rightarrow I \in \boldsymbol{O} b_{j} B / I, \beta: C \rightarrow I \in \boldsymbol{O} b j B / I$. Then $\gamma: P(u) \rightarrow \alpha=P(Y) \rightarrow D \in M$ or $B / I, \delta: P(u) \rightarrow \beta=P(Y) \rightarrow C \in M$ or $B / I$, which satisfies diagram commuting, that is, $\gamma=f \circ \delta$. In total category $T / T_{P}(I)$ on functor $P / I$, there exist two objects $s: X \rightarrow T_{P}(I) \in \boldsymbol{O} b j T / T_{P}(I), t: f^{*}(X) \rightarrow T_{P}(I) \in \boldsymbol{O} b j T / T_{P}(I)$, we have $g: u \rightarrow s=Y \rightarrow \quad X \in M \operatorname{or} T / T_{P}(I)$. Then there exists a unique morphism $k: u \rightarrow t=Y \rightarrow f^{*}(X)$ satisfying diagram commuting $g=f_{x}^{\downarrow} \circ k$. By Definition $1 f_{x}^{\downarrow}$ is a Cartesian arrow of $f$ on functor $P / I$, so if $P$ is a fibration, then the functor $P / I$ is also a fibration.

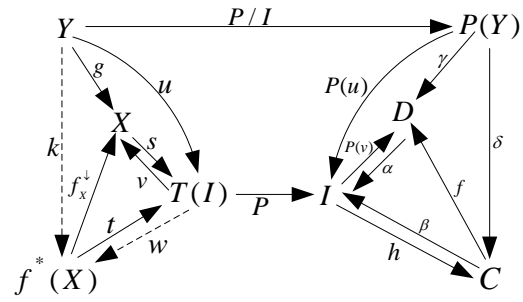

Figure 5. Cartesian Morphism $f_{x}^{\downarrow}$ of $P / I$ above $f$

Let $m: Z \rightarrow T_{P}(I) \in \boldsymbol{O} b j T / T_{P}(I)$ be an object in total category $T / T_{P}(I)$. Then $P / I(m)=\alpha$ by the definition of functor $P / I$. Let $f_{\downarrow}{ }^{2}: Z \rightarrow{ }^{\prime} f(Z)$ be an opposite Cartesian arrow of $f$ on $P$ (Figure 6). The diagram commutes $\alpha=\beta^{\circ} f$ in slice category $B / I$, there exists an unique morphism $n: " f(Z) \rightarrow T_{P}(I)$ in total category $T / T_{P}(I)$ on functor $P / I$, which satisfies diagram commuting $m=n^{\circ} f_{\downarrow}{ }_{\downarrow}$. By Definition $4 f_{\downarrow}{ }^{2}$ is an opposite Cartesian arrow of $f$ on functor $P / I$, namely, if $P$ is an opposite fibration, then the indexed functor $P / I$ is also an opposite fibration.

Therefore, if $P$ is a fibration or bifibration, the single-sorted indexed functor $P / I$ is also a fibration or bifibration. 


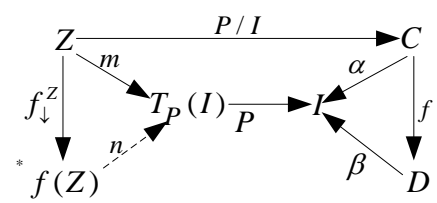

Figure 6. Opposite Cartesian Morphism $f_{\downarrow}{ }_{\downarrow}$ of ${ }_{P} / I$ Above $f$

Theorem 3 proves that indexed fibration $P / I$ and fibration $P$ have the same properties of fibration or bifibration, and also presents a construction method of indexed fibration. In fact, change of base of $P$ along domain functor $d o m: B / I \rightarrow B$ can construct a indexed fibration $P / I: T / T_{P}(I) \rightarrow B / I$. For $\forall \alpha: C \rightarrow I \in \boldsymbol{O} b j B / I$, the fiber $T_{c}$ above $C$ on $P$ is isomorphism up to the fiber $(T / T(I))_{\alpha}$ above $\alpha$ on $P / I$ [15]. And if $P$ has truth functor, then the indexed fibration $P / I$ constructed by $P$ has also truth functor.

For $\forall \alpha: C \rightarrow I \in \boldsymbol{O}_{b_{j} B} / I$, let two pullbacks of $\alpha$ along itself be $i$ and $j$ respectively. Then the product object $\alpha \times \alpha$ is $\alpha^{\circ} i$ or $\alpha^{\circ} j$, that is, product object $B / I$ in slice category is determined by its pullbacks. Similarly to Definition 11, the following is definition of relation fibration of indexed fibration $P / I$.

Definition 13. Let $P / I: T / T_{P}(I) \rightarrow B / I$ be an indexed fibration, base category $B / I$ has products. Let $\Delta / I: B / I \rightarrow B / I$ be a diagonal endo-functor in slice category $B / I$. Then $\Delta / I$ maps $\forall \alpha \in B / I$ to product object $\alpha \times \alpha$. The pullback of $P / I$ along $\Delta / I$ constructs a fibration $\operatorname{Rel}(P / I): \operatorname{Rel}\left(T / T_{P}(I)\right) \rightarrow B / I, \operatorname{Rel}(P / I)$ is relation fibration of $P / I$.

For an object $R \in O \operatorname{Obj} \operatorname{Rel}\left(T / T_{P}(I)\right)$ above $\alpha$ on the relation fibration $\operatorname{Rel}(P / I)$, object $R^{\prime} \in \boldsymbol{O} b j T / T_{P}(I)$ above $\alpha \times \alpha$ on $P / I$, and object $R^{\prime \prime} \in \boldsymbol{O} b j T$ above $\operatorname{dom}(\alpha \times \alpha)$ on $P$, there exists isomorphism $R \cong R^{\prime} \cong R^{\prime \prime}$ in [15]. The action function of $\alpha$ on natural transformation $\delta / I: I d_{B / I} \rightarrow \Delta / I$ is $(\delta / I)_{\alpha}: C \rightarrow \operatorname{dom}(\alpha \times \alpha)$, then the intuitional meaning of natural transformation $\delta / I$ is a morphism from an object to another object in slice category $в / I$. Similarly, for Definition 12, the following is the definition of equation functor of indexed fibration $P / I$.

Definition 14. Let $P: T \rightarrow B$ be a bifibration satisfying Beck-Chevalley condition between two small categories $T$ and $B, P$ has truth functor, and base category $B$ has product. Let $T_{P / I}$ be a truth functor of indexed fibration $P / I$. Then $E q_{P / I}={ }^{*}(\delta / I)^{\circ} T_{P / I}: B / I \rightarrow \operatorname{Rel}\left(T / T_{P}(I)\right)$ is an equation functor of $P / I$.

Equation functor $E q_{P / I}$ maps object $\alpha: C \rightarrow I$ in slice category $B / I$ to a unique morphism " $(\delta / I)_{\alpha}{ }^{\circ} T_{P / I}(C) \rightarrow T_{P}(I)$ above $\alpha \times \alpha$. The following is the quotient functor constructed by indexed fibration $P / I$.

\subsection{Quotient Functor and Its Lifting Equation-Preserving}

The truth functor $T_{P}: B \rightarrow T$ of fibration $P: T \rightarrow B$ is substituted by the equation functor $E q_{P}: B \rightarrow \operatorname{Rel}(T)$ of $P$, and $P$ is also substituted by its relation fibration $\operatorname{Rel}(P)$, then by Theorem 3 we construct a new fibration $\operatorname{Rel}(P) / I: \operatorname{Rel}(T) / E q_{P}(I) \rightarrow B / I$. For $\forall R \in O$ obj $\operatorname{Rel}(T), \operatorname{Rel}(P) / I$ maps $\alpha: R \rightarrow E q_{P}(I)$ to $\alpha^{\prime}: Q R \rightarrow I, \alpha$ 'is the transpose of $\alpha$ for ad joint functor $Q \dashv E q_{P}$. 
Definition 15. Let the ad joint functor $\tau \dashv \sigma: \operatorname{Rel}\left(T / T_{P}(I)\right) \rightarrow \operatorname{Rel}(T) / E q_{P}(I)$ satisfies diagram commuting, that is, $\operatorname{Rel}(P / I)=\operatorname{Rel}(P) / I{ }^{\circ} \tau$ and $\operatorname{Rel}(P) / I=\operatorname{Rel}(P / I)^{\circ} \sigma$. If $\operatorname{Rel}(P) / I$ has a right ad joint functor $E q_{(P / I)}$ such that $E q_{(P / I)}=\tau{ }^{\circ} E q_{P / I}$, then $\operatorname{Rel}(P) / I^{\circ} \tau \dashv \sigma^{\circ} E q_{(P / I)}$, we call $\operatorname{Rel}(P) / I^{\circ} \tau$ is quotient functor of indexed fibration $P / I$. Write $\operatorname{Rel}(P) / I^{\circ} \tau$ for $Q_{P / I}$, and $Q_{P / I} \dashv E q_{P / I}$.

Let $\forall R=(C, D) \in O \operatorname{obj} \operatorname{Rel}\left(T / T_{P}(I)\right)$. Then $Q_{P / I}(C, D)=C$ (Fig.7), and $\Pi(C, D)=D$. For an object in the slice category, namely, $f: D \rightarrow T_{P}(I) \in \boldsymbol{O} b j T / T_{P}(I), P / I(f)=P(D) \rightarrow I$, and $g: C \rightarrow I \in O b j B / I, \Delta / I(g)=g \times g$, namely, $\operatorname{dom}(g \times g)=P(D)$.

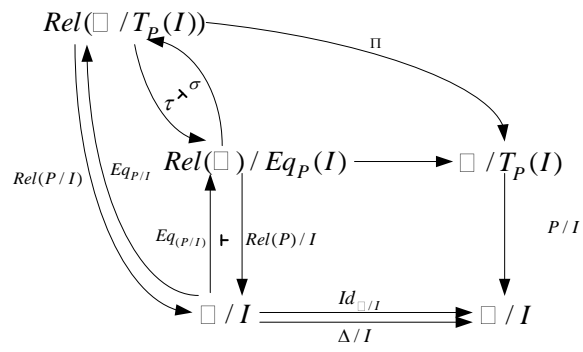

Figure 7. Construction of Quotient Functor $Q_{p / 1}$

Definition 16. Let $P: T \rightarrow B$ be a bifibration satisfying Beck-Chevalley condition with truth functor $T_{P}$ between two small categories $T$ and $B$, base category $B$ has products and pullbacks, and $P / I: T / T_{P}(I) \rightarrow B / I$ be an indexed fibration of $P$. We construct relation fibration $\operatorname{Rel}(P / I)$ of $P / I$, and equation functor $E q_{P / I}$ and quotient functor $Q_{P / I}$ of $P / I$. Let $F$ be an endo-functor in base category $B / I$ on $\operatorname{Rel}(P / I), F^{+}$be an endo-functor in total category $\operatorname{Rel}\left(T / T_{P}(I)\right)$ on the relation fibration $\operatorname{Rel}(P / I)$. If it satisfies the diagram commuting $\operatorname{Rel}(P / I)^{\circ} F^{\perp}=F^{\circ} \operatorname{Rel}(P / I)$, and the following isomorphism holds, i.e., $E q_{P / I}{ }^{\circ} F \cong F^{\perp}{ }^{\circ} E q_{P / I}$ and $F{ }^{\circ} Q_{P / I} \cong Q_{P / I}{ }^{\circ} F^{\perp}$, then $F^{\perp}$ is a lifting equation-preserving of $F$ about $\operatorname{Rel}(P / I)$ in total category $\operatorname{Rel}\left(T / T_{P}(I)\right)$.

\subsection{Semantic Behavior of Indexed Co-inductive Data Type}

For $\forall \alpha: C \rightarrow I \in O$ oj $B$ /I , a $F$-coalgebra $(\alpha, r: \alpha \rightarrow F(\alpha))$ is constructed by the action of endo-functor $F, \alpha$ is called to be carrier. The morphism between $(\alpha, r)$ and another $F$ coalgebra $(\beta, t: \beta \rightarrow F(\beta))$ is a morphism $f: \alpha \rightarrow \beta$ between their carriers, which satisfies diagram commutes, that is, $t^{\circ} f=F(f)^{\circ} r . F$-coalgebra category is constituted by $F$ coalgebra and their morphisms, denoted as Coalg $_{F}$. If the terminal $F$-coalgebra $(v F$, out $: v F \rightarrow F(v F))$ exists, it is up to a unique isomorphism with universal property determined by terminal coalgebra. The universal property is main tool for us to study semantic behavior of co-inductive data type.

Indexed co-inductive data type $v F$, as the carrier of terminal $F$-coalgebra, is maximal fixed point of functor $F$. The functor $F$ denotes syntax destructor of $v F$, and its morphism out observes semantic behaviors of $v F$ from outside during its syntax destructing. Applying equation functor $E q_{P / I}$ of indexed fibration $P / I$ to map $F$-coalgebra $(\alpha, r)$ to a $F^{\perp}$ coalgebra $E q_{P / I}(\alpha, r)=\left(E q_{P / I}(\alpha), E q_{P / I}(r): E q_{P / I}(\alpha) \rightarrow E q_{P / I}(F(\alpha)) \cong F^{\perp}\left(E q_{P / I}(\alpha)\right)\right)$. 
Accordingly, $E q_{P / I}(v F)$ is the carrier of terminal $F^{\perp}$-coalgebra, namely, equation functor $E q_{P / I}$ preserves terminal objects.

Write $\operatorname{Coalg}\left(E q_{P / I}\right)$ for functor from $F$-coalgebra category $\operatorname{Coalg}_{F}$ to $F^{\perp}$-coalgebra category Coalg $_{F^{\perp}}$, it maps objects and morphisms in base category $B / I$ on relation fibration $\operatorname{Rel}(P / I)$ to those correspondingly in total category $\operatorname{Rel}\left(T / T_{P}(I)\right)$ by equation functor $E q_{P / I}$. Therefore, functor $\operatorname{Coalg}\left(E q_{P / I}\right)$ establishes relationship between $\operatorname{Coalg}_{F}$ and Coalg $_{F^{\perp}}$ further.

Let $\left(E q_{P / I}(v F)\right.$, out $\left.{ }^{\perp}: E q_{P / I}(v F) \rightarrow F^{\perp}\left(E q_{P / I}(v F)\right)\right)$ be a terminal $F^{\perp}$-coalgebra in total category $\operatorname{Rel}\left(T / T_{P}(I)\right)$ on relation fibration $\operatorname{Rel}(P / I)$. Then $o u t^{\perp}$ is a homomorphism image of out by the action of functor $\operatorname{Coalg}\left(E q_{P / I}\right)$, that is, $\operatorname{Coalg}\left(E q_{P / I}\right)(o u t)=o u t^{\perp}$. Terminal property of terminal $F^{\perp}$-coalgebra ensures that out is up to unique isomorphism, whose existence provides extremely convenience for analyzing accurately semantic behavior of coinductive data type.

Similar to Coalg $\left(E q_{P / I}\right)$, write Coalg $\left(Q_{P / I}\right)$ for the functor from $\operatorname{Coalg}_{F^{\perp}}$ to Coalg $_{F}$. Then we have $\operatorname{Coalg}\left(Q_{P / I}\right) \dashv \operatorname{Coalg}\left(E q_{P / I}\right)$ by the ad joint property of ad joint functor in [9]. For each $\quad F^{\perp} \quad$-coalgebra $\quad\left(\omega, q: \omega \rightarrow F^{\perp}(\omega)\right) \quad, \quad \omega: X \rightarrow T_{P}(I) \in O b j \quad \operatorname{Rel}\left(T / T_{P}(I)\right) \quad$, $\operatorname{Coalg}\left(Q_{P / I}\right)(q)=Q_{P / I}(\omega) \rightarrow Q_{P / I}\left(F^{\perp}(\omega)\right) \cong F\left(Q_{P / I}(\omega)\right)$, that is, $\operatorname{Coalg}\left(Q_{P / I}\right)(q)=Q_{P / I}(q)$. Then $Q_{P / I}(q)$ is a homomorphism image of $q$ by the action of functor $\operatorname{Coalg}\left(Q_{P / I}\right)$, seen if from Figure 8. If $g: \omega \rightarrow E q_{P / I}(\alpha)$ is a $F^{\perp}$-coalgebra morphism from $q$ to $E q_{P / I}(r)$, then the $F$-coalgebra morphism $h: Q_{P / I}(\omega) \rightarrow \alpha$ from $Q_{P / I}(q)$ to $r$ is $F$-coalgebra homomorphism above $g$. Similarly, $g$ is a $F^{\perp}$-coalgebra homomorphism above $h$.

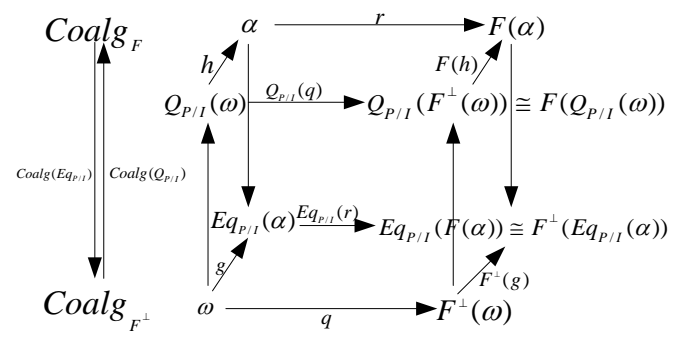

Figure 8. Ad-joint Properties of $\operatorname{Coalg}_{\left(E q_{p / 1}\right)}$ and $\operatorname{Coalg}_{\left(Q_{p / 1}\right)}$

The left ad joint $\operatorname{Coalg}\left(Q_{P / I}\right)$ of functor $\operatorname{Coalg}\left(E q_{P / I}\right)$ makes an intuitional inter-deducible relation between $F$-coalgebra $Q_{P / I}(\omega)$ as its carrier and $F^{\perp}$-coalgebra $\omega$ as its carrier, which provides a succinct and consistent modelling method for analyzing semantic behavior of indexed co-inductive data type, $v F$ as carrier of terminal coalgebra.

\subsection{Example Analysis}

Example 1. The element type of stream or infinite sequence is designated by index $I$, such as natural number $N a t$, integer Int and character Char , $\forall I \in \boldsymbol{O}_{b j} B$. For any stream $\alpha: S \rightarrow I \in \boldsymbol{O} b j B / I$, we can construct an endo-functor $F: \alpha \rightarrow I \times \alpha$ on $B / I$, where 
head $: \alpha \rightarrow I$ is head function of this stream, and tail $: \alpha \rightarrow \alpha$ is tail function erased head element. For any stream property $R \in O \operatorname{bj} \operatorname{Rel}\left(T / T_{P}(I)\right)$ in total category $\operatorname{Rel}\left(T / T_{P}(I)\right)$ on relation fibration $\operatorname{Rel}(P / I)$ of indexed fibration $P / I$, such as bi-simulation, then for another stream object $\beta: S^{\prime} \rightarrow I$ in $B / I$, a co-induction of $\alpha$ and $\beta$ on bi-simulation property $R$ is established:

Object $R$ is relation of bi-simulation between two streams $\alpha$ and $\beta$, iff $\forall(\alpha, \beta) \in R$, head $(\alpha)=\operatorname{head}(\beta)$, and $(\operatorname{tail}(\alpha), \operatorname{tail}(\beta)) \in R$.

Let the stream Stream $(I)$ be carrier $v F$ of terminal $F$-coalgebra $(v F$, out $: v F \rightarrow F(v F))$ in base category $B / I$. For each $F$-coalgebra $(\alpha, r: \alpha \rightarrow F(\alpha))$, it is lifted to a $F^{\perp}$-coalgebra $\left(X, q: X \rightarrow F^{\perp}(X)\right)$ by relation fibration $\operatorname{Rel}(P / I)$, which satisfies diagram commuting, i.e., $F \circ \operatorname{Rel}(P / I)(X)=\operatorname{Rel}(P / I)^{\circ} F^{\perp}(X)$.

Example 1 presents some fibrational tools including indexed fibration, equation functor and quotient functor to analyze deeply semantic behavior of stream by Fibrations theory, which set well math foundation for researching semantics computing and program logics of program languages.

\section{Related Works}

As a co-inductive data type with stronger ability of semantic computation, indexed coinductive data type whose math foundation is coalgebra introduces some tools such as terminality and bi-simulation to type theory, has particular advantages in analyzing and describing dynamic semantic behavior of program languages. From the situation of literature retrieval, Hagino is the earliest scholar who researched relationship between inductive and coinductive data type systematically by dialgebras structure in [16], whose works laid the foundation of co-inductive data type, but there exist certain drawbacks in polymorphism type system, co-induction application of programming and so on.

Joint efforts of many scholars promote the development of co-inductive data type further. Nogueira studied relationship between inductive and co-inductive data type and their application in polymorphism programming by method of bi-algebra in [17]. Literature [18] melted organically inductive and co-inductive data type by $\lambda$ bi-algebra and distributive laws further, it discussed the relation of syntax construction and dynamic behavior of data type. Poll extended the works of Hagino based on sub-type and inheritance, analyzed their connections of inductive and co-inductive data type by dual properties of algebra and coalgebra in [19]. Greiner etc. brought co-inductive principle in program languages, deeply discussed co-inductive data type in programming [1-20], the fruits above solved questions aforementioned to some extent. Meanwhile, in the application respects of co-inductive data type, Gimenez explored some applications by Coq which is a formal theory proof tool in [21]; Vene researched co-inductive data type in functional program language Haskell [22]. Most of fruits in hand researched relation of inductive and co-inductive data type and their applications in programming by methods of category theory and coalgebra, and those based on Fibrations theory focused on program logic reasoning of co-inductive data type and validations of co-inductive rules only. For example, Hermida and Jacobs proved co-inductive rule of terminal coalgebra with quotient type in [9], Ghani etc. broke the limitation of polynomial functor based on [9], extended their works to general functor [15].

Existing researches mainly focus on co-inductive data type, but studying for indexed co-inductive data type is still in a preliminary stage currently. There exist plenty of unsolved problems in semantic computing and program logic, especially analyzing semantic behavior. This paper explores semantic behavior of indexed co-inductive data type systematically and deeply by Fibrations theory. Compared with traditional methods including algebra and category theory, the advantages of this paper are as follows: it analyzes semantic behavior of indexed co-inductive data type succinctly by Fibrations 
theory, improves abilities of processing and proving of program languages for semantic behavior of indexed co-inductive data type; it does not depend particular computing circumstance such as domain theory and set category, construct coalgebra categories to analyze semantic behavior of indexed co-inductive data type. Those works provide solid math foundations and succinct and uniform descriptive ways for semantic computation and program logic of program languages.

\section{Conclusions}

This paper modelled on slice category $B / I$, dealt with well semantic behavior analysis and co-inductive rule description of single-sorted indexed co-inductive data type which takes $I$ as its index. But the index $I$ only points at some particular singlesorted indexed co-inductive data type; it is difficult to manage more complex manysorted indexed co-inductive data type such as mutual recursive. Therefore, extending discrete index object $I$ of single-sorted indexed fibration to indexed category $C$ to construct many-sorted fibration, representing many-sorted indexed co-inductive data type in $B$ by index set $o_{b j} C$, modelling semantic behavior of many-sorted indexed coinductive data type in indexed category $C$ by fibration $G: B \rightarrow C$, selecting different program logics aiming at different indexes are our future research work.

\section{Acknowledgements}

This work is funded by Guangdong Provincial Natural Science Foundation, China (Grant No. S2013010015944), Guangdong Provincial University Outstanding Young Teacher Programming, China (Grant No. YQ2014155), Guangdong Provincial strategic emerging industries core technology research projects, China (Grant No. 2012A010701011, 2012A010701003), Guangzhou municipal science and technology plan, China (Grant No. 201200000034) and Shaoguan municipal science and technology plan, China (Grant No. 2013CX/K61).

\section{References}

[1] J. Greiner, "Programming with Inductive and Co-inductive types", Tech. Report CMU-CS-92-109, Pittsburgh,USA: School of Computer Science, Carnegie Mellon University, (1992) January.

[2] J. Rutten, "Universal Coalgebra: a Theory of Systems", Theoretical Computer Science, vol. 1, no. 249, (2000).

[3] X. C. Zhou and Z. M. Shu, "A Survey on the Coalgebraic Methods in Computer Science", Journal of Software, vol. 10, no. 14, (2003).

[4] M. Johnson and R. Rosebrugh, "Fibrations and Universal View Updatability", Theoretical Computer Science, no. 388, (2007).

[5] M. Johnson, R. Rosebrugh and R. J. Wood, "Lenses, Fibrations and Universal Translations", Mathematics Structure in Computer Science, vol. 22, (2012).

[6] H. Tews, "Coalgebra Method for Object-oriented Specification", Doctor Thesis, Dresden, Germany: Institute Theoretische Informatik, Technischen Universiy Dresden, (2002).

[7] N. Ghani, P. Johann and C. Fumex, "Generic Fibrational Induction", Logical Methods in Computer Science. vol. 2, no. 8, (2012).

[8] D. C. Miao, J. Q. Xi, L. Y. Jia and Y. Liu, "Formal Language Algebraic Model", Journal of South China University of Technology (Natural Science Edition), vol. 10, no. 39, (2011).

[9] C. Hermida and B. Jacobs, "Structural Induction and Coinduction in a Fibrational Setting", Information and Computation, vol. 2, no. 145, (1998).

[10] M. Barr and C. Wells, "Category Theory for Computing Science”, NewYork: Prentice-Hall, (1990).

[11] D. Pavlovic, "Predicates and Fibrations", Doctor Thesis, Utrecht, Nederland: University of Utrecht, (1990).

[12] B. Jacobs, "Categorical Logic and Type Theory", Amsterdam, Nederland: Elsevier Science, (2001).

[13] W. He, "Category Theory", Beijing: Science Press (2006).

[14] P. Morris, T. Altenkirch, "Indexed Containers", Proc. of the 24th Symp. On Logic in Computer Science, Los Angeles, California, USA, (2009).

[15] N. Ghani, P. Johann and C. Fumex. Indexed Induction and Coinduction, Fibrationally, Logical Methods in Computer Science, no. 9, (2013) ,pp. 3-6. 
[16] T. Hagino, "A Categorical Programming Language, Doctor Thesis", Edinburgh,UK: Laboratory for Foundations of Computer Science, Dept of Computer Science, University of Edinburgh, (1987)

[17] P. Nogueira and J. Moreno-Navarro, "Bialgebra Views: a Way for Polytypic Programming to Cohabit with Data Abstract", WGP' 08 Proceedings of the ACM SIGPLAN Workshop on Generic Programming, NY, USA, (2008).

[18] J. D. Su and S. S. Yu, "Coinductive Data Types and Their Applications in Programming Languages", Computer Science, vol. 11, no. 38, (2011).

[19] E. Poll, "Subtyping and Inheritance for Categorical Datatypes", RIMS Lecture Notes, no. 1023, (1998).

[20] R. Hinze, "Reasoning about Codata", Lecture Notes in Computer Science, no. 6299, (2010).

[21] E. Gimenez and P. Casteran, "A Turorial on Co-inductive Types in Coq", http: www.labri.fr/perso /casteran/RecTutorial.pdf,(1998).

[22] V. Vene, "Categorical Programming with Inductive and Coinductive Types", Doctor Thesis, Tartu, Estonia: University of Tartu, (2000).

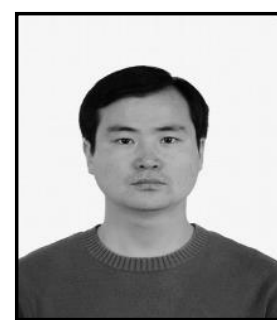

MIAO De-cheng, He received his $\mathrm{PhD}$ degree in Institute of Computer Science \& Engineering from South China University of Technology, in 2012. He is currently an associate professor in Institute of Information Science \& Engineering, Shaoguan University, China. His research interests include formal languages theory, categorical theory methods.

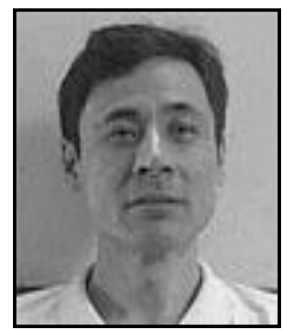

XI Jian-qing, He received his $\mathrm{PhD}$ degree in Department of Computer Science \& Technology from National University of Defense Technology, in 1992. He is currently a professor Institute of Computer Science \& Engineering from South China University of Technology, China. His research interests include software theory, database and network computation.

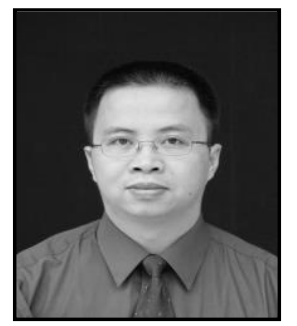

JIANG Chang-jin, He received his $\mathrm{PhD}$ degree in Institute of Computer Science \& Engineering from South China University of Technology, in 2010. He is currently an associate professor in Institute of Information Science \& Engineering, Shaoguan University, China. His research interests include natural language processing, machine learning and computer games.

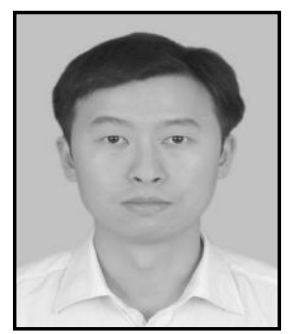

LIANG Yong-lin, He received his Master of Software Engineering in the college of Software from Huazhong University of Science and Technology. Since 2009 he is a lecturer of Shaoguan University. His current research interests include Artificial Intellegence and Intelligence Human Computer Interaction. 
International Journal of $u-$ and e- Service, Science and Technology Vol.9, No. 6 (2016) 